\title{
Krankenversicherungstarife im Spielfeld divergierender Kräfte
}

\author{
Die für Leistungserbringer geltenden KVG-Tarife sind, obwohl rechtlich geregelt, ein \\ Spielball von Ökonomie und Politik. Zu Unrecht wird vom Primat der Wirtschaftlich- \\ keit ausgegangen, entscheidend sind auch die Qualität und Wirksamkeit der Behand- \\ lung bzw. der Leistungen sowie die volkswirtschaftlichen Ersparnisse.
}

\begin{abstract}
Die Versicherten bezahlen u. a. für die Leistungen von Ärzten und Spitälern Prämien. Im Behandlungsfall stehen die Krankenkassen für das Honorar der Leistungserbringer ein. Basis sind hierbei Tarifverträge zwischen den Leistungserbringern und den Versicherern. Diese legen die Höhe des Behandlungsentgeltes fest; sie werden von den Kantonsregierungen und zum Teil vom Bundesrat genehmigt oder, im Fall einer ausbleibenden Einigung, behördlich festgelegt. Wenig überraschend steht hierbei die Tariffestlegung nicht einfach im Gutdünken der Tarifpartner oder der Regierungen; vielmehr sind gesetzliche Vorschriften zu beachten.
\end{abstract}

nahme bzw. einer Leistung ist mithin auch von der Wahl des Ziels, dem sog. Effektivitätsmass, abhängig. Erst wenn das Ziel feststeht, kann aus ökonomischer Sicht sinnvoll beurteilt werden, ob der mit einer Massnahme zu erwartende, zusätzliche Wert deren zusätzliche (Opportunitäts-)Kosten übersteigt. Im Krankenversicherungsbereich bedeutet dies, dass man sich auch Gedanken zur Wirksamkeit und Zweckmässigkeit machen muss, wenn die Wirtschaftlichkeit und Effizienz zu prüfen ist. Letztlich sind diese Aspekte alle miteinander verbunden. Eine isolierte Betrachtung führt in die Irre.

\section{Muss eine günstigere Behandlung gewählt werden, wenn mit der teureren die Arbeitsfähigkeit schneller wiederhergestellt werden kann?}

So bestimmt zum Beispiel Artikel 32 des Krankenversicherungsgesetzes, dass die obligatorische Krankenversicherung Leistungen nur zu bezahlen hat, wenn diese wirksam, zweckmässig und wirtschaftlich sind. Artikel 59c der Krankenversicherungsverordnung bloss eine Verordnungsbestimmung, kein formelles Gesetz - bestimmt sodann autoritativ für die Tarifparteien und die Behörden, dass der Tarif höchstens die transparent ausgewiesenen Kosten der Leistung und die für eine effiziente Leistungserbringung erforderlichen Kosten decken darf. Ein Wechsel des Tarifmodells darf ferner keine Mehrkosten verursachen. Der Teufel liegt, wie oft im Krankenversicherungsbereich, im Detail, und die Verhältnisse sind komplexer, als es auf den ersten Blick scheint. Dies zeigen die nachfolgenden Beispiele paradigmatisch.

So gibt es ökonomisch eine Vielzahl von Kostenbegriffen. Welche Kosten sind bei Tarifen gemeint? Was ist der Bezugsrahmen? Muss zum Beispiel eine günstigere Behandlung gewählt werden, auch wenn mit der teureren vielleicht die Arbeitsfähigkeit schneller wiederhergestellt werden kann? Die Effizienz einer Mass-
Die gelebte Realität sieht derzeit anders aus. Im Rahmen von Tariffestsetzungen muss sich auch der Eidgenössische Preisüberwacher äussern. Für diesen steht gemäss dem Preisüberwachungsgesetz die Dimension der Wirtschaftlichkeit absolut im Zentrum. Behörden und Gerichte folgen in der Regel seinen Überlegungen, weil er eine Fachstelle ist. Basis sind hierbei Wirtschaftlichkeitsüberlegungen, während weitere Aspekte auf der Strecke bleiben. Ausgegangen wird von der stillschweigenden, gesundheitsökonomisch und rechtlich unzulässigen Annahme, dass alle zugelassenen Leistungserbringer die Anforderungen des Gesetzes bezüglich der Wirksamkeit und Zweckmässigkeit gleichermassen erfüllen. Indes: Es gibt wirksame und weniger wirksame Behandlungen, gute und schlechte Spitäler, wirtschaftliche und weniger wirtschaftliche Ärzte. Dies reflektiert sich nicht in der Rechtsprechung.

Bei den Tariffestsetzungen zeigt sich sodann einmal mehr, wie problematisch die Mehrfachrolle der Kantonsregierungen im Krankenversicherungsbereich ist. Sie sind für die Umsetzung des Zulassungsstopps 
verantwortlich. Sie betreiben in ihrer Funktion als Spitaleigentümer in vielen Kantonen Spitäler und Polikliniken und subventionieren diese teilweise. Sie sind also Partei. Trotzdem legen sie die Tarife bei Streitigkeiten zwischen den Tarifpartnern verbindlich fest - vor dem Hintergrund ihrer Interessenlage zumeist zulasten des ambulanten Bereichs sowie von privaten stationären Anbietern. Daher hat für das Bundesverwaltungsgericht die Meinung der Kantonsregierungen bei tariflichen Festlegungen weniger Gewicht als die Auf-

\section{Eine dringende Forderung: In Tarifverfahren muss das Bundesverwaltungsgericht Wirksamkeit und Zweck- mässigkeit überprüfen}

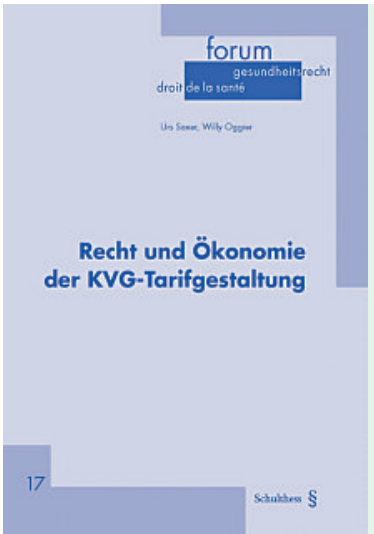

\section{Neuerscheinung: Recht und Ökonomie der KVG-Tarifgestaltung}

Die Autoren des hier veröffentlichten Artikels setzen sich in einem eben erschienenen Buch umfassend mit derselben Thematik auseinander.

Urs Saxer, Willy Oggier. Recht und Ökonomie der KVG-Tarifgestaltung. Eine kritische Würdigung, unter besonderer Berücksichtigung von Art. 59c KV. forum gesundheitsrecht Band 17. Zürich: 2010; 112 Seiten, broschiert. ISBN 978-3-7255-6117-9. 48 CHF. auch eine Systematik zu entwickeln, wie der Krankheitszustand (die Morbidität) und die Veränderung der Morbidität im Rahmen der Tarifentscheide berücksichtigt werden können. In diesem Punkt geht es nicht nur um eine Qualitäts-, sondern auch um eine MengenKomponente, dürfte doch davon auszugehen sein, dass bei steigender Morbidität auch mehr Leistungen durch die Leistungserbringer auszuführen sind.

Mit der Tariffestsetzung hängt auch die Frage zusammen, ob Leistungserbringer in einer Sozialversicherung Gewinne machen dürfen. Klar ist, dass Leistungserbringer für ihre Leistungen zu entgelten sind, aber - zumindest nach den Tarifgrundsätzen - höchstens für die transparent ausgewiesenen und für eine effiziente Leistung erforderlichen Kosten. Was heisst dies? Müssen auch Verluste in Kauf genommen werden? Gesundheitsökonomisch hängt dies vor allem von den konkreten «Märkten» ab. Besteht ein Überangebot, sollten vor allem bei vergleichbarer Wirksamkeit hocheffiziente Leistungserbringer Gewinne machen können. Besteht dagegen Unterversorgung, müsste idealtypisch jeder wirksame und zweckmässige Leistungserbringer Aussicht auf Gewinn haben, wenn er seine Leistungen einigermassen effizient ausführt. Damit bekommen weitere Leistungserbringer einen Anreiz, in diesem von Unterversorgung geprägten Umfeld tätig zu werden. Funktioniert dieser Anpassungsprozess, dann reduzieren sich auch die Gewinnaussichten mit zunehmendem Abbau der vorhandenen Unterversorgung.

Diese wenigen Überlegungen zeigen, wie verwickelt das Verhältnis zwischen Ökonomie, Recht und Politik im Krankenversicherungsbereich ist. Das Gesetz verwendet ökonomische Begriffe mit nicht eindeutigem Inhalt und statuiert gleichzeitig zu erreichende Ziele, welche teilweise gegenläufig sind. Die Exekutiven entscheiden auch nach politischen Gesichtspunkten. Die Gerichte wiederum verweisen auf den Preisüberwacher. Damit wird die Tariffestsetzung zum Spielball divergierender Kräfte und teilweise gegenläufiger Interessen. Klarere Konzepte, klarere Begriffe und eine bessere rechtsstaatliche Durchdringung sind die Desiderata an die Politik. Von besonderer Bedeutung dürfte dabei eine morbiditätsorientierte Verbesserung des Risikoausgleichs sein, wie sie auch der neue Bundesrat Didier Burkhalter bereits mehrmals erwähnt hat. Sie könnte hier eine positive Veränderung der Anreize bewirken. 